\title{
The Be-test in the Li-rich star \#1657 of NGC 6397: evidence for Li-flash in RGB stars? *
}

\author{
L. Pasquini ${ }^{1}$, A. Koch $^{2}$, R. Smiljanic ${ }^{3}$, P. Bonifacio ${ }^{4}$, and A. Modigliani ${ }^{1}$ \\ ${ }^{1}$ ESO - European Southern Observatory, Karl-Schwarzschild-Strasse 2, 85748 Garching bei München, Germany \\ e-mail: lpasquin@eso.org \\ 2 Zentrum für Astronomie der Universität Heidelberg, Landessternwarte, Königstuhl 12, 69117 Heidelberg, Germany \\ 3 Department for Astrophysics, Nicolaus Copernicus Astronomical Center, ul. Rabiańska 8, 87-100 Toruń, Poland \\ ${ }^{4}$ GEPI, Observatoire de Paris, CNRS, Univ. Paris Diderot, Place Jules Janssen, 92190 Meudon, France
}

Received 9 December 2013 / Accepted 16 January 2014

\section{ABSTRACT}

\begin{abstract}
Context. The Li-rich turn-off star recently discovered in the old, metal-poor globular cluster NGC 6397 could represent the smoking gun for some fundamental, but very rare episode of Li enrichment in globular clusters and in the early Galaxy.

Aims. We aim to understand the nature of the Li enrichment by performing a spectroscopic analysis of the star, in particular of its beryllium $(\mathrm{Be})$ abundance, and by investigating its binary nature.

Methods. We used the VLT/UVES spectrograph to observe the near UV region where the Be II resonance doublet and the NH bands are located. We also re-analyzed an archival Magellan/MIKE spectra for $\mathrm{C}$ and $\mathrm{O}$ abundance determination.

Results. We could not detect the Be II lines and derive an upper limit of $\log (\mathrm{Be} / \mathrm{H})<-12.2$ that is consistent with the Be observed in other stars of the cluster. We could detect a weak G-band, which implies a mild carbon enhancement $[\mathrm{C} / \mathrm{Fe}]+0.4 \pm 0.2$. We could not detect the UV NH band, showing that this star is less $\mathrm{N}$-enhanced than other stars of the cluster, and we derive an upper limit $[\mathrm{N} / \mathrm{Fe}]<0.0$. For oxygen we could not convincingly detect any of the near UV OH lines, which implies that oxygen cannot be strongly enhanced in this star. This is consistent with the detection of the strongest line of the O I triplet at $777 \mathrm{~nm}$, which is contaminated by telluric absorptions but is consistent with $[\mathrm{O} / \mathrm{Fe}] \sim 0.5$. Combining the UVES and Mike data, we could not detect any variation in the radial velocity greater than $0.95 \mathrm{kms}^{-1}$ over 8 years.

Conclusions. The chemical composition of the star strongly resembles that of "first generation" NGC 6397 stars, with the huge Li as the only deviating abundance. Not detecting Be rules out two possible explanations of the Li overabundance: capture of a substellar body and spallation caused by a nearby type II SNe. Discrepancies are also found with respect to other accretion scenarios, except for contamination by the ejecta of a star that has undergone the RGB Li-flash. This is at present the most likely possibility for explaining the extraordinary Li enrichment of this star.
\end{abstract}

Key words. stars: abundances - globular clusters: individual: NGC 6397 - stars: atmospheres

\section{Introduction}

Rare, peculiar objects in the Milky Way halo and in globular cluster (GCs) can be extremely interesting, because they may be smoking guns for understanding phenomena that occurred in the early Galaxy. The extremely Li-rich star \#1657 in NGC 6397 (三2MASS J17410651-5342290) is one of these objects. Lithium is a complex element because it can be created in a number of ways such as Big Bang nucleosynthesis, in the cool bottom burning process during the asymptotic giant branch (AGB) phase, or via spallation. On the other hand, it is easily destroyed in the stellar interiors. In the context of GCs, $\mathrm{Li}$ is fundamental for constraining cosmological models, understanding the role of diffusion, and explaining the nature of the multiple populations now clearly detected in most clusters (see Korn 2012, for a review).

NGC 6397 is one of the best-studied GCs. It is metalpoor $([\mathrm{Fe} / \mathrm{H}] \sim-2 \mathrm{dex})$ and has been age-dated through its Beryllium abundance and stellar evolutionary models (Pasquini et al. 2004). In this metallicity range, warm stars around the turn off (TO) display a constant lithium abundance, irrespective of their temperatures, around $\log (\mathrm{Li} / \mathrm{H})+12=2.2$, the so-called "Spite plateau" (Spite \& Spite 1982). Extensive spectroscopic

* Based on observations collected at ESO, VLT, Chile, Proposal 091.D-0198(A). surveys have shown that turn-off (TO) stars in NGC 6397 have a Li abundance very close to the "Spite plateau" (Thévenin et al. 2001; Bonifacio et al. 2002; Lind et al. 2009; González Hernández et al. 2009), and a debate is going on about whether the Li abundance around the TO shows the signature of diffusion (Korn et al. 2006).

Star \#1657 is located at the cluster TO. While it is fully representative of the remainder of the GC giant and TO stars in terms of metallicity, $\alpha$-element abundance ratios, and stellar parameters, it has a $\mathrm{Li}$ abundance about 100 times higher than any other NGC 6397 stars. With an exceptional abundance of $\log \left({ }^{7} \mathrm{Li} / \mathrm{H}\right)+12=4.2$ (assuming non-LTE), it is one of the stars with the highest Li ever observed (Koch et al. 2011, 2012), and only very few other dwarf or giant stars show comparable levels of enhancement (Reyniers \& Van Winckel 2001; Deliyannis et al. 2002; Monaco et al. 2012; Adamów et al. 2012). What produced such a high Li abundance in a TO star of a GC? Considering that the primordial abundance of Li produced in the Big Bang is almost 100 times lower than the one observed in \#1657, some extra production and contamination mechanism must be invoked, either by engulfing substellar objects (see, e.g., Pasquini et al. 2007), or by the mass transfer from a more massive star that went through an evolved phase, nurturing the manufacturing of extra-Li. The best contenders for the latter are AGB 
stars (Cameron \& Fowler 1971) or the acting of the cool bottom process (CBP) on the red giant branch (RGB; Sackman \& Boothroyd 1999). Alternatively, Li could have been produced by the explosion of a nearby supernova $(\mathrm{SN})$ through spallation (cf., e.g., Smiljanic et al. 2008).

All of these processes have some serious shortcomings, as discussed in Koch et al. (2011, 2012). However, one key test can be performed to easily distinguish the main scenarios, viz. the beryllium test. Amongst the different hypotheses, the mass transfer from an evolved companion (of any kind) cannot add any $\mathrm{Be}$ to the star, since Be is easily destroyed in the stellar interiors. On the other hand, either the engulfing of a substellar companion or the spallation produced by a nearby SN explosion would enhance $\mathrm{Be}$ in the atmosphere of the presently observed star by a large factor.

The engulfing of a substellar companion with the same $(\mathrm{Li} / \mathrm{Be})$ ratio as in the primordial GC material would enhance the $\mathrm{Be}$ abundance by a factor of $\sim 65$ with respect to the original $\mathrm{Be}$ of the star (i.e., the same overenhancement as seen for Li). When there is an energetic, near-by event, such as the explosion of a super- or hypernova, the enhancement factor can reach as high as $\sim 5600$ (assuming a ratio of $\mathrm{Li} / \mathrm{Be}=4.2$, by number, in the spallation material as suggested by the hypernova models of Nakamura \& Shigeyama 2004).

We consider that, as for other GCs, NGC 6397 shows evidence of multiple populations, both in its color-magnitude diagram (di Criscienzo et al. 2010) and in chemical abundance space, with stars having the same metallicity but different light element abundances (e.g., Carretta et al. 2009; Lind et al. 2011; Gratton et al. 2012, and references therein). In this context, Shen et al. (2010) demonstrate for the more metal-rich GC NGC 6752 that the first-generation stars must have enriched some of the second-generation stars in Li. Similarly, in NGC 6397, Pasquini et al. (2008) find a Li-rich but O-poor star, which also requires some pollution with $\mathrm{Li}$ by a prior population.

The present work aims to investigate the Be abundance and that of other elements, to better characterize the properties of this star.

\section{Observations and data reduction}

The observations were obtained with the UVES spectrograph at the VLT (Dekker et al. 2000). The bluest setup, centered at $346 \mathrm{~nm}$ was used to cover the UV part of the spectrum, together with the red setup centered at $580 \mathrm{~nm}$. Seven blue observations of 1.5 hours each were requested and executed in the period of April 14 - June 18, 2013. For each UV observation, two red observations were acquired. Two observations were repeated because they were hampered by poor conditions, so the total number of observations consists of 17 red and 9 blue spectra. The blue slit was set at a width of 1.1", yielding a resolving power of $R \sim 38000$, while the red slit width was selected at $0.6^{\prime \prime}$, corresponding to $R \sim 67000$.

The spectra were reduced using the ESO reflex pipeline (Larsen et al. 2008; Ballester et al. 2011). To gather a sufficiently high signal-to-noise ratio $(\mathrm{S} / \mathrm{N})$ in the extreme $\mathrm{UV}$, where the Be lines are located (around $313 \mathrm{~nm}$ ), the blue spectra were obtained with the detector in binned mode. The extracted, wavelength-calibrated spectra were corrected for earth motion and finally combined. Out of the available nine blue spectra, two revealed a $\mathrm{S} / \mathrm{N}$ that was too low to be used, and one was contaminated by a very strong, high-energy event (cosmic ray) right in the region of the Be lines, and they have not been used. The
Table 1. Radial velocities for star \#1657 (first two columns), measured by two methods, and the contaminant.

\begin{tabular}{lccc}
\hline \hline \multirow{2}{*}{ Date of obs. } & \multicolumn{3}{c}{$v_{\mathrm{HC}}\left[\mathrm{km} \mathrm{s}^{-1}\right]$} \\
\cline { 2 - 4 } & Li 670.7 nm & CCF & Contaminant \\
\hline 20130427 & 19.37 & 20.2 & -101.2 \\
20130429 & 19.06 & 18.8 & -102.8 \\
20130527 & 19.26 & $\ldots$ & -101.2 \\
20130528 & 19.07 & $\ldots$ & -102.6 \\
20130615 & 19.39 & 19.9 & -101.8 \\
20130616 & 18.80 & $\ldots$ & -102.2 \\
\hline
\end{tabular}

Notes. See text for details.

remaining six spectra were combined to form the resulting spectrum used in the Be analysis of star \#1657.

\section{Analysis}

\subsection{Radial velocity}

As already noted by Koch et al. (2011), the spectrum of star \#1657 is contaminated by the flux of a second star with a very different radial velocity. We have used two ways of estimating radial velocities: for the GC TO primary, we directly used the Doppler-shift of the strong and deep $\mathrm{Li}$ resonance line at $670.7 \mathrm{~nm}$. For the contaminating foreground companion, we cross-correlated the lower part of the red spectrum with a template of a solar-type star (Melo et al. 2001).

In the resulting cross-correlation function $(\mathrm{CCF})$, the peak of the contaminant is very pronounced, indicating that this object is much more metal rich than a typical member star of NGC 6397. The peak of star \#1657 itself is also often present in the CCF, but with a weak and more uncertain CCF peak, when compared to the direct measurement of the Li line. Moreover, the Li line is visibly strong and measurable in all spectra, so that we adopt these measurements for the remainder of the paper.

The radial velocity variability of the GC star from the Li line on the 17 exposures is $350 \mathrm{~m} \mathrm{~s}^{-1}(1 \sigma)$ and thus perfectly compatible with instrumental and centering instabilities. Exposures taken over the same night show a $1 \sigma$-scatter of only $220 \mathrm{~m} \mathrm{~s}^{-1}$, and these have been averaged in Table 1 .

Figure 1 shows the radial velocity measurements of the two components when combining the previous observations obtained with the Magellan/MIKE instrument (Koch \& McWilliam 2011; Koch et al. 2011, 2012) with our new ones by UVES. To aid the readability of the velocity curves, we split the time axis so as to shorten the gaps of up to six years between the observing runs.

We note that there are systematic differences in the observational setups and in the data analysis of the MIKE and UVES observations that could lead to potential biases. It is then striking that there is no evidence of any significant, systematic velocity variation within the combined data. In fact, we could not find any suitable orbital solution that would describe the observed velocity data of either component. We conclude that the star does not show any radial velocity variations larger than $0.95 \mathrm{~km} \mathrm{~s}^{-1}$ $(1 \sigma)$ over eight years of observations, and its velocity is in perfect agreement with the estimated mean systemic velocity of the cluster (Milone et al. 2006).

\subsection{Be abundances}

We adopt the stellar parameters derived by Koch et al. (2011), namely $T_{\text {eff }}=6282 \pm 250 \mathrm{~K}, \log g=4.1 \pm 0.2 \mathrm{dex}, \xi=$ $1.2 \pm 0.8 \mathrm{~km} \mathrm{~s}^{-1}$, and an iron abundance, measured from the 
L. Pasquini et al.: The Be-test in the Li-rich star \#1657 of NGC 6397: evidence for Li-flash in RGB stars?

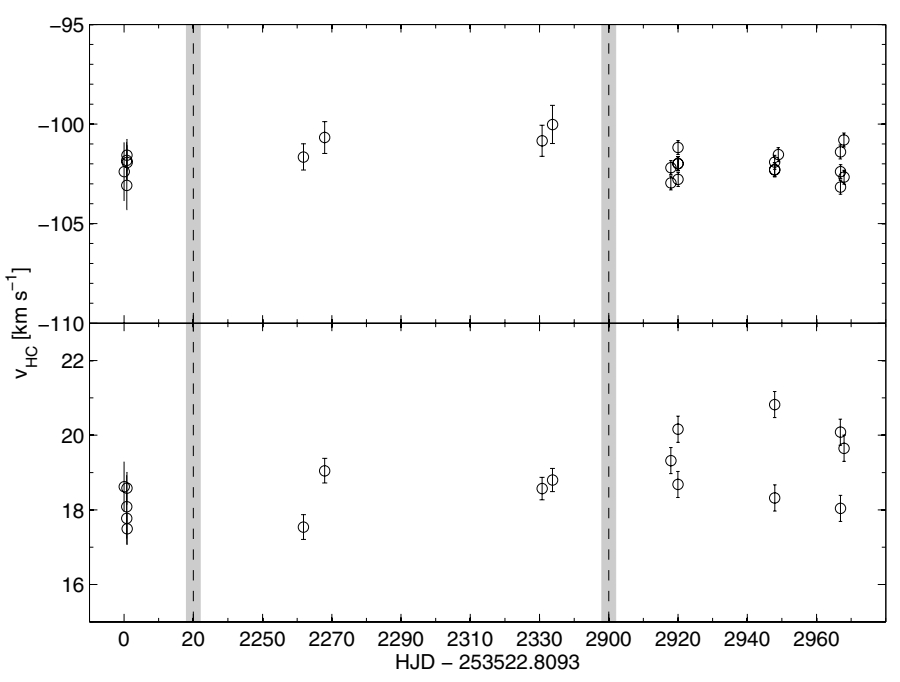

Fig. 1. Combined radial velocity of star \#1657 (bottom panel) and the metal-rich foreground contaminant (top panel). The segmented range of the $x$-axis covers more than 8 years of observations. The vertical bars separate the three observing runs.

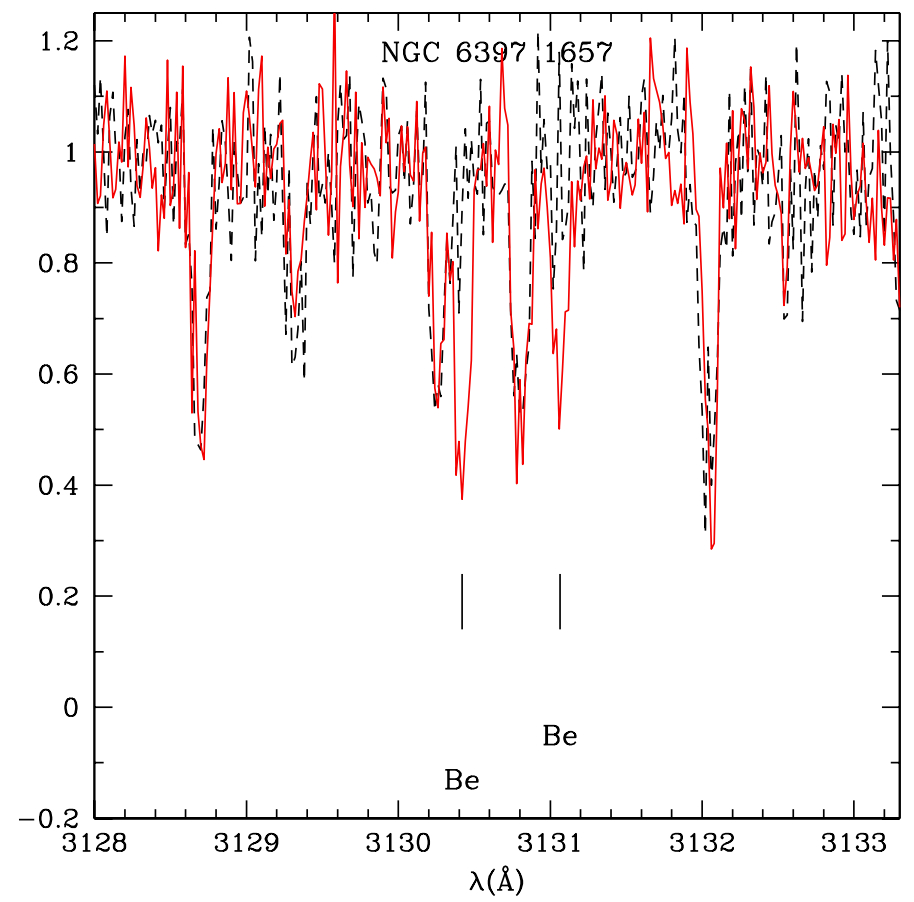

Fig. 2. Be-lines in \#1657. The observed spectrum is shown as black, dashed line. The red line is a synthetic spectrum with $\mathrm{Be} / \mathrm{H}=-11.5$, to which noise has been added. Both Be lines are not detected and only a conservative upper limit could be set at $\log (\mathrm{Be} / \mathrm{H})=-12.2$.

neutral species, of $[\mathrm{Fe} / \mathrm{H}]=-1.93 \pm 0.06$ (stat.) \pm 0.18 (sys.). The Be doublet region is shown in Fig. 2, with the synthetic spectrum overimposed, computed as in Smiljanic et al. (2011). The stellar spectrum has a low $S / N \sim 10$ and shows no clear detection of the Be lines. We determine an upper limit of $\log (\mathrm{Be} / \mathrm{H})=$ -12.2 by performing Monte Carlo simulation on synthetic spectra with different abundances and trying to detect the Be II lines by basic statistics (average counts, smallest flux pixel, highest flux pixel). This upper limit is compatible with the Be observed in the other two TO stars of NGC 6397 for which this line has been detected (Pasquini et al. 2004). A strong conclusion is that star \#1657 does not show any evidence of Be enhancement with respect to stars of the same cluster or with respect to field stars with similar metallicity.

When assuming the linear relation derived in Smiljanic et al. (2009) between $[\mathrm{Fe} / \mathrm{H}]$ and $\log (\mathrm{Be} / \mathrm{H})$, star \#1657 should have $\log (\mathrm{Be} / \mathrm{H}) \sim-12.8$. Assuming that the $\mathrm{Li}$ enhancement is due to pollution, that initial $\mathrm{Li}$ should be $A(\mathrm{Li})=2.20$ (Spite \& Spite 1982; Lind et al. 2009, González Hernández et al. 2009), and that the polluting material had normal $\mathrm{Be} / \mathrm{Li}$ composition (equivalent to $A(\mathrm{Li})=2.20$ and $\log (\mathrm{Be} / \mathrm{H}) \sim-12.8)$, the star should have accreted an amount of Be that would increase its chemical composition to $\log (\mathrm{Be} / \mathrm{H}) \sim-11.5$. Assuming the Be abundance from the field star regression $(\log (\mathrm{Be} / \mathrm{H})=-12.8)$ as reference value is a conservative assumption, because this value is lower than what is observed in the TO stars of the cluster.

\section{3. $\mathrm{C}, \mathrm{N}$, and $\mathrm{O}$}

The UVES spectra do not cover any region that can be used to determine the carbon abundance. The Mike spectra cover the G-band, albeit at low S/N. In such warm dwarfs the G-band is very weak, but it is clearly detected. We conclude that carbon is mildly enhanced in this star: $[\mathrm{C} / \mathrm{Fe}]=0.4 \pm 0.2$.

An excellent diagnostic is provided for nitrogen by the UV $\mathrm{NH}$ band and $336 \mathrm{~nm}$, whicht has been used by Pasquini et al. (2008) to measure $\mathrm{N}$ in three TO stars in this cluster. To our surprise we were unable to detect the band in star \#1657, whose spectrum in the $\mathrm{NH}$ band region is distinctly different from that of star A228, which has very similar atmospheric parameters and for which Pasquini et al. (2008) measured $[\mathrm{N} / \mathrm{H}]=-0.75$. In fact, all three stars in this clusters measured by Pasquini et al. (2008) show similar $\mathrm{N}$ enhancement with respect to iron. We estimate $[\mathrm{N} / \mathrm{H}]<-2.0$, both from comparison with synthetic spectra and differentially with respect to star A228.

To search diagnostics for the oxygen abundance we inspected the UV spectra for the presence of $\mathrm{OH}$ lines (see González Hernández et al. 2010), but we were not able to detect any. The same is true for the three stars observed by Pasquini et al. (2008), this allows us to rule out a strong enhancement in oxygen in this star, with respect to other stars in the cluster. This result is also consistent with the detection of only the strongest line of the O I triplet at $777.1 \mathrm{~nm}$ in the Mike spectrum. We have a very uncertain estimate of the equivalent width of about $1.9 \mathrm{pm}$, which corresponds to $[\mathrm{O} / \mathrm{Fe}] \sim 0.5$ when taking the NLTE corrections for oxygen into account, according to the prescriptions of Shen et al. (2010). The line is possibly contaminated by telluric absorption on its red wing. Comparison of the line in this star with other TO stars of the same cluster observed under the same conditions with MIKE shows the strength of the O I triplet in star \# 1657 to be quite typical.

\subsection{Sodium}

Koch \& McWilliam (2011) obtained $\alpha$-element abundance ratios of three red giants and two TO stars in NGC 6397 and could measure Na-abundances in the three red giant stars of their sample. Upon revisiting the MIKE spectra, here we also measured equivalent widths (EWs) of the near-infrared Na line at $819.5 \mathrm{~nm}$ of the TO stars. As a result, we obtain $[\mathrm{Na} / \mathrm{Fe}]$ ratios of -0.03 and -0.40 dex for the two regular (i.e., Li-normal) TO stars and, for the Li-rich \#1657, an upper limit of $[\mathrm{Na} / \mathrm{Fe}]<-0.56$, based on an EW of $<1.6 \mathrm{pm}$. We can use this to associate the giants and one of the TO stars with the second generation of stars in the GC, characterized by elevated Na- and depleted $\mathrm{O}$ abundances 
Table 2. Elemental abundances for star \#1657 and for typical firstgeneration NGC 6397 star.

\begin{tabular}{lll}
\hline \hline Element & $\# 1657$ & NGC 6397 \\
\hline$[\mathrm{Fe} / \mathrm{H}]$ & $-1.93 \pm 0.2$ & -2.02 \\
$\mathrm{Li} / \mathrm{H}$ & 4.2 & $2.1 / 2.2^{d}$ \\
$\mathrm{Be} / \mathrm{H}$ & $<-12.2$ & $-12.2 /-12.3^{a}$ \\
{$[\mathrm{C} / \mathrm{Fe}]$} & $0.4 \pm 0.2$ & $<0.5^{b}$ \\
{$[\mathrm{~N} / \mathrm{H}]$} & $<-2$ & $-3 /-1^{b, c}$ \\
{$[\mathrm{O} / \mathrm{Fe}]$} & $\sim+0.5$ & $+0.4 /+0.6^{b}$ \\
{$[\mathrm{Na} / \mathrm{Fe}]$} & $<-0.56$ & $-0.35 /-0.65^{d}$ \\
\hline
\end{tabular}

References. ${ }^{(a)}$ Pasquini et al. (2004); (b) Carretta et al. (2005); ${ }^{(c)}$ Pasquini et al. (2008); ${ }^{(d)}$ Lind et al. (2009) .

(Carretta et al. 2009). The very low $[\mathrm{Na} / \mathrm{Fe}]$ ratios in the remaining TO star and the present \#1657 suggests that they are part of the first stellar generation of NGC 6397, which formed from material that was not yet enriched with Na-rich ejecta coming from p-capture reactions in early, massive AGB-polluters (e.g., Decressin et al. 2007; D’Ercole et al. 2008).

\section{Discussion}

Table 2 summarizes the abundances measured in star \#1657, compared with the value range observed in first-generation NGC 6397 stars. Exceptions are obviously Li overabundance and possibly $\mathrm{N}$ underabundance. The abundance of $\mathrm{N}$ in TO stars of NGC 6397 seems highly variable and apparently independent of any other light element abundance. Pasquini et al. (2008) find high $\mathrm{N}$ in three TO stars irrespective of their light element abundances, and Carretta et al. (2005) show that N can span almost two orders of magnitude among NGC 6397 subgiants, with two $\mathrm{N}$-poor stars among the richest in $\mathrm{O}$, but all span a very narrow $\mathrm{C}$ abundance range. If we assume an analogy with field stars, we expect a low $\mathrm{N}$ abundance for the first generation of cluster stars. Chemically therefore star \#1657 has no signature of pollution from processed material; in contrast all its values point toward an NGC 6397 first-generation star, with the exception of Li.

Before the present study, Koch et al. (2011, 2012) discussed five possible causes of the Li- pollution for star \#1657: (i) capture of a substellar body; (ii) type II Supernovae; (iii) diffusion; (iv) contamination by an AGB companion; (v) contamination from an RGB companion having undergone cool bottom processing.

As far as spallation, planet engulfment, and internal mixing/diffusion are concerned, the first two can be excluded by the regular abundance of Be. Internal mixing and diffusion can be excluded because of the extremely high abundance of $\mathrm{Li}$ observed. The two last processes implying pollution from an evolved companion remain, in principle, viable.

If $\mathrm{Li}$ has been produced in a companion then our radial velocity measurements coupled with those of Koch et al. (2011) imply that the orbit is highly inclined or has a very long period (hundreds or thousands of years) and that the companion star should now be subluminous (e.g., a white dwarf). Alternative explanations could be that the binary has since been disrupted, e.g. due to collisions or tidal effects, or that the accretion took place during a close encounter of two unbound stars. Of course it cannot be excluded that the stars formed from a polluted gas, therefore not only the external atmosphere composition has been altered. This would solve the problem of the missing companion, but would imply an enormous amount of $\mathrm{Li}$, because in this case the whole mass of the star would have been affected.

Pollution through the winds of a companion that passed through a (super-) AGB phase, during which the extra-Li would have been produced through hot bottom burning (Cameron \& Fowler 1971; Ventura \& D’Antona 2011) is in principle a possibility. In the lower mass regime, this could lead to elevated levels of s-process elements, which is not observed in the Li-rich star \#1657 (Koch et al. 2011). However, the absence of significant s-process-rich material cannot be unambiguously used as an argument against AGB contamination, since that material will not be dredged-up from deeper layers in the more massive (6-8 $\left.M_{\odot}\right)$ super-AGB stars (Ventura \& D'Antona 2010). Nevertheless, a super-AGB star should also produce sizeable amounts of the light elements $\mathrm{Na}$ and $\mathrm{K}$, none of which are seen in \#1657. This means that we can reject the hypothesis of the extra lithium in this star originating in a super-AGB envelope.

Pollution by an RGB star does not contradict the observations at our disposal, if one would admit the presence of a "Li flash" in RGB stars. Lithium-rich giants are known in the Galaxy (de la Reza 1996), and they present in external dwarf galaxies at the rate of $1 \%$ (Kirby et al. 2012). Like star $\# 1657$, they seem to only be enriched in Li. They possibly represent a short phase of stellar evolution, followed by a Li flash (de la Reza et al. 1996). No RGB star with such high Li content has been discovered so far, and even if several propositions exist in the literature (Denissenkov \& Weiss 2000, Palacios et al. 2001), no detailed mechanism has been proven for such a flash. But in first approximation, this would fit with the absence of enhanced $\mathrm{Na}$, the low $\mathrm{N}$, and the composition of a first-generation cluster stars not showing evidence of binarity. In this sense it would be different from what produces the well-known light elements anti-correlations in globular clusters. The high abundance of $\mathrm{C}$ observed may contradict the trend of diminishing $\mathrm{C}$ abundance along the RGB (Carretta et al. 2005), but given that we do not know when and how the hypothetical Li flash occurs, this discrepancy may not be so stringent. Also, the sudden extra mixing invoked to explain the Li flash is of a substantially different timescale than the slow extra mixing invoked along the RGB to explain the slow decay of $\mathrm{C}$ with luminosity. We finally notice that, if $\mathrm{Be} / \mathrm{H}$ was confirmed at a level comparable to the other NGC 6397 stars (Pasquini et al. 2004), then the RGB pollution hypothesis should also be dropped, since Be has already been diluted in the atmospheres of evolved stars.

Exploring the RGB hypothesis further, de la Reza et al. (1997) argue that, at a certain point in the life of a giant, an abrupt mixing mechanism can lead to a rapid surface injection of material with fresh internal ${ }^{7} \mathrm{Be}$, which is rapidly transformed to ${ }^{7} \mathrm{Li}$. The same mechanism would then enable the formation of a circumstellar shell of gas and dust that is later ejected in the interstellar medium. Denissenkov \& Herwig (2004) show that extra mixing, driven by faster rotation, is able to produce a Li flash. They argue that such a spinning could be produced in close binaries or by engulfing a massive planet. Modeling the Li flash is beyond the scope of this paper. We note, however, that an alternative source of extra mixing could come from the possible coupling between shells and cores in giants, given the recent finding by the Kepler satellite that cores of RGB stars rotate $\sim 13$ times faster than the envelopes (Beck et al. 2012; Deheuvels et al. 2012). The core rotation is faster than what is expected by solid rotation, but slower than expected if shellular rotation is assumed, indicating that core-envelope coupling is present at a certain level. 
Although the presence of a Li-O correlation, as present in NGC 6752, has not been established in NGC 6397, we should expect a very large oxygen abundance if such a relation existed and if the extremely high Li abundance in star \#1657 was linked to the phenomenon producing that correlation. However, a strong enhancement in $\mathrm{O}$ is firmly ruled out by our observations.

Exotic explanations, such as Li production around compact objects, which could explain the high Li abundances observed in low-mass X-ray binaries (see, e.g., Guessoum \& Kazanas 1999) seem unlikely, since Casares et al. (2007) favor the simpler Lipreservation scenario due to tidal locking rather than the Li production in these systems for the case of Cen-X4 .

The $\mathrm{Li}$ abundance in \#1657 is most likely not directly linked to the spread in $\mathrm{Li}$ abundances observed in NGC 6397 and other GCs, but is linked to a very exceptional event. Contamination by an RGB that went through the Li-flash so far seems the most likely possibility. Regardless of the explanation, it shows that local production of $\mathrm{Li}$ is possible in GCs, with peaks well exceeding the cosmic values. This work reinforces the concept that extreme care should be used when inferring the properties of primordial nucleosynthesis based on observations of $\mathrm{Li}$ in GCs not only because of the clear presence of second-generation stars that show light elements correlations and that can be Li polluted, but also because even stars that can be considered bona fide first-generation may be polluted in Li, the RGB Li-flash was a common phenomenon. If every RGB star underwent the Liflash, as argued by de la Reza et al., we could predict that the absolute Li abundance and its spread among GC TO stars (even selecting only first-generation stars) should be higher than what is observed among field stars, because of the presence of "disguised" second-generation stars that, like star \#1657, have been only contaminated by the ejecta of RGB stars.

Acknowledgements. L.P. acknowledges the Visiting Researcher (PVE) program of the CNPq Brazilian Agency, at the Federal University of Rio Grande do Norte, Brazil. A.K. acknowledges the Deutsche Forschungsgemeinschaft for funding from Emmy-Noether grant Ko 4161/1. P.B. acknowledges support from the Conseil Scientifique de l'Observatoire de Paris and from the Programme National de Cosmologie et Galaxies of the Institut National des Sciences de l'Univers of CNRS. R.S. is supported by the National Science Centre of Poland through grant 2012/07/B/ST9/04428.

\section{References}

Adamów, M., Niedzielski, A., Villaver, E., Nowak, G., \& Wolszczan, A. 2012, ApJ, 754, L15

Ballester, P., Bramich, D., Forchi, V., et al. 2011, in Astronomical Data Analysis Software and Systems XX, ASP Conf. Proc., 442, 261

Beck, P.G., Montalban, J., Kallinger, T., et al. 2012, Nature, 481, 55
Bonifacio, P., Pasquini, L., Spite, F., et al. 2002, A\&A, 390, 91

Cameron, A. G. W., \& Fowler, W. A. 1971, ApJ, 164, 111

Carretta, E., Bragaglia, A., Gratton, R. G., et al. 2009, A\&A, 505, 117

Casares, J., Bonifacio, P., González Hernández, J. I., Molaro, P., \& Zoccali, M. 2007, A\&A, 470, 1033

Decressin, T., Meynet, G., Charbonnel, C., Prantzos, N., \& Ekström, S. 2007, A\&A, 464, 1029

Dekker, H., D’Odorico, S., Kaufer, A., Delabre, B., \& Kotzlowski, H. 2000, Proc. SPIE, 4008, 534

de La Reza, R., Drake, N. A., \& da Silva, L. 1996, ApJ, 456, L115

de La Reza, R., Drake, N. A., da Silva, L., Torres, C. A. O., \& Martin, E. L. 1997, ApJ, 482, L77

Deheuvels, S., Garcia, R. A., Chaplin, W. J., et al. 2012, ApJ, 756, 19

Deliyannis, C. P., Steinhauer, A., \& Jeffries, R. D. 2002, ApJ, 577, L39

Denissenkov, Pavel, A., \& Weiss, A. 2000 A\&A, 308, 773

Denissenkov, Pavel, A., \& Herwig, F. 2004, ApJ, 612, 1081

D’Ercole, A., Vesperini, E., D’Antona, F., McMillan, S. L. W., \& Recchi, S. 2008, MNRAS, 391, 825

di Criscienzo, M., D’Antona, F., \& Ventura, P. 2010, A\&A, 511, A70

González Hernández, J. I., Bonifacio, P., Caffau, E., et al. 2009, A\&A, 505, L13

González Hernández, J. I., Bonifacio, P., Ludwig, H.-G., et al. 2010, A\&A, 519, A46

Gratton, R., Sneden, C., \& Carretta, E. 2004, ARA\&A, 42, 385

Gratton, R. G., Carretta, E., \& Bragaglia, A. 2012, A\&ARv, 20, 50

Guessoum, N., \& Kazanas, D. 1999, ApJ, 512, 332

Koch, A., \& McWilliam, A. 2011, AJ, 142, 63

Koch, A., Lind, K., \& Rich, R. M. 2011, ApJ, 738, L29

Koch, A., Lind, K., Thompson, I. B., \& Rich, R. M. 2012, Mem. Soc. Astron. It. Suppl., 22, 79

Korn, A. J. 2012, Mem. Soc. Astron. It. Suppl., 22, 64

Korn, A. J., Grundahl, F., Richard, O., et al. 2006, Nature, 442, 657

Kirby, E. N., Fu, X., Guhathakurta, P., \& Deng, L. 2012 ApJ, 725, L16

Larsen, J. M., Ballester, P., D’Odorico, V., et al. 2008, in 2007 ESO Instrument Calibration Workshop (Berlin, Heidelberg: Springer Verlag), 403

Lind, K., Primas, F., Charbonnel, C., Grundahl, F., \& Asplund, M. 2009, A\&A, 503,545

Lind, K., Charbonnel, C., Decressin, T., et al. 2011, A\&A, 527, A148

Melo, C.., Pasquini, L., \& de Medeiros, J. R., 2001, A\&A, 375, 851

Milone, A. P., Villanova, S., Bedin, L. R., et al. 2006, A\&A, 456, 517

Monaco, L., Villanova, S., Bonifacio, P., et al. 2012, A\&A, 539, A157

Nakamura, K., \& Shigeyama, T. 2004, ApJ, 610, 888

Palacios, A., Charbonnel, C., \& Forestini, M. 2001, A\&A, 375, L9

Pasquini, L., Bonifacio, P., Randich, S., Galli, D., \& Gratton, R. G. 2004, A\&A, 426, 651

Pasquini, L., Döllinger, M. P., Weiss, A., et al. 2007, A\&A, 473, 979

Pasquini, L., Ecuvillon, A., Bonifacio, P., \& Wolff, B. 2008, A\&A, 489, 315

Reyniers, M., \& Van Winckel, H. 2001, A\&A, 365, 465

Sackmann, I.-J., \& Boothroyd, A. I. 1999, ApJ, 510, 217

Shen, Z.-X., Bonifacio, P., Pasquini, L., \& Zaggia, S. 2010, A\&A, 524, L2

Smiljanic, R., Pasquini, L., Primas, F., et al. 2008, MNRAS, 385, L93

Smiljanic, R., Pasquini, L., Bonifacio, P., et al. 2009, A\&A, 499, 103

Smiljanic, R., Randich, S., \& Pasquini, L. 2011, A\&A, 535, A75

Spite, F., \& Spite, M. 1982, A\&A, 115, 357

Thévenin, F., Charbonnel, C., de Freitas Pacheco, J. A., et al. 2001, A\&A, 373, 905

Ventura, P., \& D'Antona, F. 2010, MNRAS, 402, L72

Ventura, P., \& D'Antona, F. 2011, MNRAS, 410, 2760 